\title{
Emerging Impact of Skeletal Muscle in Health and Disease
}

\author{
Roger A. Fielding $\cdot$ Stuart H. Ralston • \\ Rene Rizzoli
}

Published online: 17 February 2015

(c) Springer Science+Business Media New York 2015

It has been over 60 years since Huxley first described the essential force transmitting properties of voluntary striated skeletal muscle [1]. At no time since then has the importance of skeletal muscle integrity been more pronounced. Although skeletal muscle comprises $40-50 \%$ of total body mass, this tissue has been relatively understudied compared to brain, liver, cardiac, bone, and other tissues. Despite the fact that skeletal muscle is necessary for locomotion, oxygen consumption, whole-body energy metabolism, and substrate turnover and storage, a relative lack of attention has been paid to this tissue that is essential for many daily functions and activities [2].

Robust skeletal muscle mass is essential for maintaining whole-body homeostasis and health [3]. With advancing age, there is a loss of skeletal muscle mass and function that contributes to declines in physical functioning, increased disability, and mortality [4]. It is with this background that we decided to devote this issue of Calcified Tissues and Musculoskeletal Research International to the subject of skeletal muscle in health and disease. This issue follows on the renaming of the journal to better attract

\footnotetext{
R. A. Fielding $(\bowtie)$

Nutrition, Exercise Physiology, and Sarcopenia Laboratory, Jean Mayer USDA Human Nutrition Research Center on Aging at Tufts University, 711 Washington Street, Boston, MA 02111, USA

e-mail: roger.fielding@tufts.edu
}

\section{S. H. Ralston}

Rheumatic Diseases Unit, University of Edinburgh, Edinburgh, UK

\section{R. Rizzoli}

Bone Disease Service, Faculty of Medicine, University of Geneva Hospital, Geneva, Switzerland papers that deal with other components of the musculoskeletal system, including skeletal muscle.

Skeletal muscle fibers possess a unique phenotype. The structural organization of the contractile proteins, actin, myosin, and troponin are closely aligned with the sarcoplasmic reticulum which regulates calcium signaling and the energy-transducing mitochondria. Frontera and Ochala elegantly describe the major structural components of the contractile proteins, the role of calcium homeostasis in controlling muscle force generation, and the interaction of the contractile elements with the muscle's extensive metabolic capacity.

Skeletal muscle fibers demonstrate a unique highly regulated plasticity that adapts to external stimuli including circulating hormones (reviewed by Sipila), nutrient supply (reviewed by Margolis), and loading conditions as reviewed by Marcotte et al. This article highlights the intracellular signaling pathways that are necessary to induce skeletal muscle growth in vivo and how these pathways are modulated under varying loading conditions. Both the traditional $\mathrm{mTORC} 1$ and more novel beta2 signaling pathways are discussed as putative activators of muscle hypertrophy. Following on this, Margolis and Rivas highlight the interactive effects of adequate nutrition and exercise training on skeletal muscle homeostasis and discuss the central molecular pathways involved in these adaptations.

Like bone, skeletal muscle mass and function is regulated by a number of circulating hormones. Interestingly, skeletal muscle does contain active estrogen receptors, and Sipila in her review has addressed the role of circulating estrogens on skeletal muscle structure and function. This further highlights the synergistic role of the circulating estrogens on both bone and skeletal muscle.

Important breakthroughs in our understanding of the required signaling pathways necessary for muscle growth 
have catalyzed the development of several novel therapeutic agents including selective androgen receptor modulators, myostatin antagonists, and troponin activators. Many of these compounds are currently being evaluated in Phase II and III clinical in specific patient populations with deficits in neuromuscular function. In their review, Meriggioli and Roubenoff have reviewed the current therapeutic targets of these pro-myogenic compounds and discussed the status of current pharmacological development in this area.

Finally, the series closes with a discussion about the interaction of the muscle-bone unit. The review by Girgis has highlighted the importance of targeting both muscle and bone in any therapeutic strategies to reduce fracture risk.

We hope that the readers of Calcified Tissues and Musculoskeletal Research International will find the skeletal muscle issue a valuable resource. We also hope this special issue encourages investigators across the spectrum of musculoskeletal research (bone, tendon, ligament, and muscle) to embrace the integrative and translational nature of these disciplines whether they work in basic or clinical science.

\section{References}

1. Huxley H, Hanson J (1954) Changes in the cross-striations of muscle during contraction and stretch and their structural interpretation. Nature 173:973-976

2. Wolfe RR (2006) The underappreciated role of muscle in health and disease. Am J Clin Nutr 84:475-482

3. Haran PH, Rivas DA, Fielding RA (2012) Role and potential mechanisms of anabolic resistance in sarcopenia. J Cachexia Sarcopenia Muscle 3:157-162

4. Fielding RA, Vellas B, Evans WJ, Bhasin S, Morley JE, Newman $\mathrm{AB}$, Abellan van Kan $\mathrm{G}$, Andrieu S, Bauer J, Breuille D, Cederholm T, Chandler J, De Meynard C, Donini L, Harris T, Kannt A, Keime Guibert F, Onder G, Papanicolaou D, Rolland Y, Rooks D, Sieber C, Souhami E, Verlaan S, Zamboni M (2011) Sarcopenia: an undiagnosed condition in older adults. Current consensus definition: prevalence, etiology, and consequences. International working group on sarcopenia. J Am Med Dir Assoc $12: 249-256$ 